\title{
Rasgos de personalidad y actitud hacia la elaboración de tesis en estudiantes del cuarto año de Psicología de la Universidad Peruana Unión - Filial Juliaca
}

\author{
Personality traits and attitude towards the development of theses seniors psychology at the \\ Universidad Peruana Union - Subsidiary Juliaca.
}

\author{
Oscar Javier Mamani Benito, Dámaris Quinteros Zúñiga²
}

\begin{abstract}
RESUMEN
Objetivo: Determinar la relación entre los rasgos de personalidad y actitud hacia la elaboración de tesis en estudiantes del cuarto año de Psicología de la Universidad Peruana Unión - Filial Juliaca. Material y métodos: De enfoque cuantitativo, diseño no experimental, corte transversal y nivel descriptivo-correlacional. La población estuvo conformada por 39 estudiantes. Se aplicó el inventario de personalidad NEO PI-R forma S, y una escala de actitud hacia la elaboración de tesis. Resultados: Existen correlaciones entre el rasgo apertura y actitud hacia la elaboración de tesis $(\mathrm{r}=0.537, \mathrm{p}<0.05)$, y entre el rasgo conciencia y actitud hacia la elaboración de tesis $(\mathrm{r}=510$, $\mathrm{p}<0.05)$; así también no se halló correlación entre el rasgo neuroticismo y actitud hacia la elaboración de tesis ( $\mathrm{r}=$ $-0.010, \mathrm{p}>0.05)$, entre el rasgo extraversión y actitud hacia la elaboración de tesis $(\mathrm{r}=0.407, \mathrm{p}>0.05)$, y entre el rasgo agradabilidad y actitud hacia la elaboración de tesis $(r=-0.261, \mathrm{p}>0.05)$.
\end{abstract}

Palabras clave: Actitud, personalidad, tesis.

\begin{abstract}
Objective: to determine the relationship between personality traits and attitude towards developing thesis in the fourth year students of psychology at the Peruvian Union University - Subsidiary Juliaca. Material and Methods: a quantitative approach, no experimental design, cross-sectional, descriptive correlational level. The population consisted of 39 students. Personality inventory NEO PI-R form S, and a scale of attitude towards developing thesis was applied. Results: there are correlations between : the trait openness and attitude towards the development of thesis $(\mathrm{r}=0.537, \mathrm{p}<0.05)$, and between the trait awareness and attitude towards the development of thesis $(r=510, p<0.05)$; well no correlation between neuroticism trait and attitude towards the development of thesis $(r=-0.010, p>0.05)$ between the extraversion trait and attitude towards the development of thesis $(r=0.407, p>0.05)$ were found and between agreeableness trait and attitude towards the development of thesis $(r=-0.261, p>0.05)$.
\end{abstract}

Keywords: Attitude, personality, research. 


\section{INTRODUCCIÓN}

La relevancia de la investigación científica, en el proceso de formación de los estudiantes, radica en que promueve en ellos habilidades cognitivas, características del pensamiento divergente y creativo. Es por eso que la tesis de grado, como investigación científica, ha marcado pauta importante para el progreso alcanzado por la civilización en el ámbito social, económico, cultural, sanitario, político, tecnológico e innovativo (Rietveldt y Vera, 2012).

Al respecto de la realidad en América Latina, en opinión de Ángel et al. (2010), la productividad científica es muy baja en comparación a los países desarrollados, especialmente la investigación estudiantil enfrenta grandes dificultades. En el Perú, si se toma como indicador el número de publicaciones científicas, en relación con la población del país, la producción científica generada en el país $(0,21$ artículos publicados por millón de habitantes en el año 2007) es inferior a la de otros países de América Latina, como Chile (2,14 publicaciones científicas por millón de habitantes ese mismo año), y está muy por debajo de los países líderes en la ciencia, como los Estados Unidos de América (12,86 por millón de habitantes ese mismo año) (Albornoz, 2009).

Estos datos dejan en evidencia lo señalado por Góngora (2002), quien manifiesta que la universidad peruana y sus respectivos estudios de pre y posgrado se encuentran en una profunda crisis en cuanto a investigación científica; del mismo modo, San Martín y García (2006) refieren que una de las causas de la crisis de la investigación estudiantil en el Perú deriva de que la tesis, como investigación científica y como actividad académica previa a la titulación en la carrera universitaria, ha tenido diversas connotaciones negativas con el transcurrir de los años, pues de ser un requisito para el bachillerato pasó a ser uno de los principales requisitos de la titulación llegando, en la actualidad, a ser relegada como una de las varias modalidades para obtener el título universitario.

En un esfuerzo por abordar este fenómeno, se han realizado diversas investigaciones tanto a nivel nacional como internacional. La mayoría de investigadores han abordado esta problemática mediante el estudio de las actitudes hacia la investigación (Jaimes, 1998; Sánchez, y otros, 2000; Verástegui, 2002; Valverde, 2005 y Laura, 2011), así también, algunas otras han involucrado variables como la influencia de los factores personales e institucionales, siendo este el caso de las investigaciones más actuales (Parra, 2010; Uribe y Márquez, 2011 y Rietveldt y Vera, 2012).
Sin embargo, en el afán de profundizar el estudio de los factores personales, fueron Portocarrero y Bielich (2006), quienes mediante un estudio cualitativo respecto a la manera en que los estudiantes viven el proceso de elaborar una tesis, y a través de una serie de entrevistas, hallaron ciertas características psicológicas que predisponen negativamente a los alumnos que están en proceso de elaborar una tesis.

Según Meléndez (2003), cuando se habla de predisposiciones, estas pertenecen al campo de las actitudes, es por ello que toda actitud implica un proceso psicológico que permite al individuo evaluar constantemente las situaciones, hechos, lugares y objetos de su vida personal y social, asimismo, Cabrera et al. (2011) refiere que la actitud no es innata en el sujeto, sino que su aprendizaje y formación depende, en gran medida, de la complejidad cognoscitiva de cada individuo; así también Salinas (2005) asevera que en la formación de ciertas predisposiciones, denominadas actitudes, es la personalidad de cada individuo la que cumple un función fundamental en su aprendizaje y formación.

Según McCrae y Costa (1998), la personalidad se entiende como una serie de tendencias básicas y características diferenciales en cada individuo, que predisponen la conducta en todos los ámbitos de acción del ser humano. Se ha podido encontrar diversas investigaciones que relacionan los rasgos de personalidad bajo el modelo de los cinco factores (Costa y McCrae, 1992), con variables como el desempeño escolar, rendimiento académico, inteligencia y los estilos de afrontamiento; hallando una correlación significativa entre los mismos (Claux, 1998; Castro y Martina, 2001; Niño de Guzmán, Calderón, y Cassaretto, 2003; Sánchez de Tagle et al., 2010). Precisamente, son estos hallazgos los que generan la motivación para determinar la correspondencia entre las cinco grandes dimensiones de la personalidad y la variable actitud hacia la elaboración de tesis. Por lo expuesto, se tiene el objetivo de determinar la relación entre los rasgos de personalidad y la actitud hacia la elaboración de tesis de los estudiantes del cuarto año de Psicología de la Universidad Peruana Unión - Filial Juliaca.

\section{MATERIAL Y MÉTODOS}

El presente estudio pertenece a un enfoque cuantitativo, de diseño no experimental, de corte transversal y nivel descriptivo-correlacional (Hernández, Fernández, y Baptista, 2010).

La población de estudio está conformada por los estudiantes del $4 .^{\circ}$ año de Psicología (39) de la 
Universidad Peruana Unión - Filial Juliaca, año 2012; de los cuales se decidió realizar un muestreo de tipo no probabilístico. Utilizando el criterio por conveniencia se halló una muestra de 17 participantes, los cuales estaban acorde con los criterios de inclusión $\mathrm{y}$, además, superaron los criterios de exclusión.

En relación a los instrumentos, para medir la variable rasgos de personalidad, se utilizó el inventario de personalidad NEO PI-R forma S, que es un cuestionario autoaplicado que consta de 240 afirmaciones, de las cuales 106 son formuladas en forma inversa. Para cada dimensión existe un total de 48 ítemes, las 30 facetas de la prueba son contestadas por 8 ítemes cada una. Adicionalmente el inventario incluye 3 ítemes de validez mas no una escala completa de deseabilidad social o de mentiras, ya que los autores consideran que dichas escalas no han demostrado su utilidad y validez con la prueba (Costa y McCrae, 1992).

Esta prueba cuenta con una adaptación realizada en el Perú por Cassaretto (1999), con 439 estudiantes universitarios entre 17 y 21 años de edad de Lima Metropolitana. En dicho estudio se encontró que la confiabilidad para las dimensiones era entre $0,84 \mathrm{y}$ 0,89 según el alfa de Crombach, y para las facetas eran en su mayoría óptimas, pero 8 no cumplieron con un nivel aceptable; así los coeficientes alfa de Crombach de las facetas puntuaron entre 0,37 y 0,79 .
El análisis factorial de la prueba reprodujo la fórmula de los cinco factores satisfactoriamente.

Del mismo modo, para medir la variable actitud hacia la elaboración de tesis, se decidió construir un instrumento tomando como referencia investigaciones similares. Se procedió a la elaboración de una escala de actitudes, tipo Likert, la misma que se construyó en base a los presupuestos teóricos de Bautista (2011), y criterios técnico-metodológicos encontrados en Blanco y Alvarado (2005). En cuanto a la validez del instrumento, para hallar la validez de contenido se recurrió al juicio de expertos, para lo cual se contó con la colaboración de 4 profesionales especialistas en el campo de la metodología de la investigación y lingüística. Asimismo, para la validez de criterio se utilizó el coeficiente $\mathrm{V}$ de Aiken, hallando un valor de .92, con lo cual se concluye que este instrumento es válido. Asimismo, para la confiabilidad del instrumento, se procedió a realizar un estudio piloto en una población similar a la población de estudio, para lo cual se contó con 48 alumnos del $5 .^{\circ}$ año de la Facultad de Ingeniería de la Universidad Peruana Unión-Filial Juliaca. Luego del análisis estadístico, mediante el coeficiente Alfa de Cronbach, se halló el valor de .87. Para incrementar el valor de alfa se recurrió al análisis discriminatorio de ítems, por lo cual se decidió eliminar 7 ítemes. La escala final quedó compuesta por 40 ítemes, con un valor alfa de .91, lo cual indica que el instrumento es altamente confiable.

\section{RESULTADOS}

Tabla 1

Características de personalidad de los estudiantes de $4 .^{\circ}$ año de Psicología de la Universidad Peruana Unión-Filial Juliaca.

\begin{tabular}{lllllll}
\hline \multirow{2}{*}{ Dimensión } & Bajo & \multicolumn{3}{c}{ Medio } & \multicolumn{3}{c}{ Alto } \\
\cline { 2 - 7 } & $\mathrm{f}$ & $\%$ & $\mathrm{f}$ & $\%$ & $\mathrm{f}$ & $\%$ \\
\hline Neuroticismo & 4 & $23.5 \%$ & 10 & $58.9 \%$ & 3 & $17.6 \%$ \\
Extraversión & 6 & $35.3 \%$ & 7 & $41.2 \%$ & 4 & $23.5 \%$ \\
Apertura & 4 & $23.5 \%$ & 9 & $52.9 \%$ & 4 & $23.6 \%$ \\
Agradabilidad & 6 & $35.3 \%$ & 9 & $52.9 \%$ & 2 & $11.8 \%$ \\
Conciencia & 5 & $29.4 \%$ & 8 & $47.3 \%$ & 4 & $23.4 \%$ \\
\hline
\end{tabular}

Como se puede observar en la tabla 1, el 58.9\% de los evaluados presenta el rasgo neuroticismo a un nivel medio, el $41.2 \%$ presenta el rasgo extraversión a un nivel medio, el $52.9 \%$ presenta el rasgo apertura a un nivel medio, también el $52.9 \%$ presenta el rasgo agradabilidad a un nivel medio, y finalmente el $47.3 \%$ presenta el rasgo conciencia a un nivel medio. 
Rasgos de personalidad y actitud hacia la elaboración de tesis en estudiantes del cuarto año de Psicología de la Universidad Peruana Unión - Filial Juliaca

Tabla 2

Actitud hacia la tesis universitaria de los estudiantes de $4 .^{\circ}$ año de Psicología de la Universidad Peruana Unión-Filial Juliaca.

\begin{tabular}{lll}
\hline Nivel & $\mathrm{F}$ & $\%$ \\
Desfavorable & 4 & $23.5 \%$ \\
Favorable & 11 & $64.7 \%$ \\
Muy favorable & 2 & $11.8 \%$ \\
\hline
\end{tabular}

En la tabla 2 se observa que el $64.7 \%$ de los evaluados demuestra una actitud favorable hacia la elaboración de tesis, el $23.5 \%$ demuestra una actitud desfavorable, y el $11.8 \%$ demuestra una actitud muy favorable.

Tabla 3

Análisis de correlación entre los rasgos de personalidad y actitud hacia la elaboración de tesis en estudiantes del $4 .^{\circ}$ año de Psicología de la Universidad Peruana Unión-Filial Juliaca.

\begin{tabular}{lll}
\hline \multirow{2}{*}{ Dimensiones } & \multicolumn{2}{l}{ Actitud hacia la elaboración de tesis } \\
\cline { 2 - 3 } Neuroticismo & $\mathrm{R}$ & $\mathrm{P}$ \\
Extraversión & -0.010 & 0.969 \\
Apertura & 0.407 & 0.105 \\
Agradabilidad & $0.537^{\star}$ & 0.026 \\
Conciencia & -0.261 & 0.312 \\
\hline
\end{tabular}

* La correlación es significante al nivel 0,05 (bilateral).

Tal como se puede apreciar en la tabla 3, se halló una correlación positiva significativa entre el rasgo apertura y la actitud hacia la elaboración de tesis $(\mathrm{r}=0.537, \mathrm{p}<0.05)$; asimismo, se halló correlación positiva significativa entre el rasgo conciencia y actitud hacia la elaboración de tesis $(0.510, \mathrm{p}<0.05)$. Mientras que no se halló correlación entre el rasgo

\section{DISCUSIÓN}

Respecto a la relación entre los rasgos de personalidad y la actitud hacia la elaboración de tesis, se hallaron correlaciones en dos dimensiones de la personalidad, siendo estos el rasgo apertura $\mathrm{y}$ actitud hacia la elaboración de tesis $(\mathrm{r}=0.537$, $\mathrm{p}<0.05$ ), y el rasgo conciencia y actitud hacia la elaboración de tesis $(0.510, \mathrm{p}<0.05)$; así también no se hallaron correlaciones entre el rasgo neuroticismo $\mathrm{y}$ actitud hacia la elaboración de tesis $(\mathrm{r}=-0.010$, $\mathrm{p}>0.05$ ), entre el rasgo extraversión y actitud hacia la elaboración de tesis $(r=0.407, p>0.05)$, y entre el rasgo agradabilidad y actitud hacia la elaboración de tesis $(\mathrm{r}=-0.261, \mathrm{p}>0.05)$. Estos resultados concuerdan con las investigaciones de Claux (1998), quien estudió neuroticismo y actitud hacia la elaboración de la tesis $(r=-0.010, p>0.05)$, también entre el rasgo extraversión y actitud hacia la elaboración de tesis $(r=0.407, p>0.05)$, y también entre el rasgo agradabilidad y actitud hacia la elaboración de tesis $(\mathrm{r}=-0.261, \mathrm{p}>0.05)$.

la relación entre el modelo de los cinco factores y el desempeño escolar, encontrando que los rasgos apertura y conciencia son predictores del desempeño académico.

Respecto a la relación entre el rasgo neuroticismo y actitud hacia la elaboración de tesis, los resultados obtenidos demuestran que no existe correlación entre ambas $(r=-0.010, p>0.05)$, Este resultado concuerda con los hallazgos de Niño de Guzmán, Calderón y Cassaretto (2003), quienes no encontraron correlación entre el rasgo neuroticismo y el rendimiento académico. Sobre este punto, este hecho podría encontrar explicación si se toma en cuenta que lo que busca esta dimensión es evaluar la tendencia de los individuos para enfrentar de manera inadecuada 
el malestar psicológico (McCrae y Costa, 1998), entonces se haría evidente que el rasgo neuroticismo tendría mayor impacto en términos de salud mental en las personas, lo cual implicaría que de alguna manera no intervenga ni influya en el desarrollo y la formación de ciertas predisposiciones que los estudiantes manifiestan al momento de afrontar un proceso que involucra también cierto rendimiento intelectual, como el de elaborar una tesis.

Con respecto a la relación entre el rasgo extraversión y actitud hacia la elaboración de tesis, los resultados obtenidos demuestran que no existe correlación entre ambas $(\mathrm{r}=0.407, \mathrm{p}>0.05)$. Este resultado también concuerda con los hallazgos de Niño de Guzmán et al. (2003), quienes trataron de hallar correlaciones entre los rasgos de personalidad y el rendimiento académico, encontrando que no existe correlación entre el rasgo extraversión y el rendimiento académico. Sobre este punto, este hecho podría encontrar explicación si se entiende la dimensión extraversión como la cantidad e intensidad de las interacciones sociales, la necesidad de estimulación y la capacidad para experimentar placer y otras emociones positivas (McCrae \& Costa, 1998); entonces se haría evidente que el rasgo extraversión tendría más impacto en la búsqueda de interacción social y estimulación, lo cual implica que de alguna manera no intervenga ni influya en el desarrollo y la formación de ciertas predisposiciones positivas o negativas que los estudiantes manifiestan al momento de afrontar una situación como la elaboración de una tesis.

Con respecto a la relación entre el rasgo apertura y actitud hacia la elaboración de tesis, los resultados obtenidos demuestran que sí existe correlación entre ambas $(r=0.537, p<0.05)$. Este hallazgo concuerda con los resultados obtenidos por Claux (1998), también halló influencia y capacidad predictiva por parte del rasgo apertura en el desempeño escolar. Asimismo, según McCrae y Costa (1998), la dimensión apertura presenta asociaciones muy modestas con el nivel de educación y la inteligencia, incluyendo elementos como creatividad y pensamiento divergente, por lo tanto, esto tendría congruencia pues según Vieytes (2004), el proceso de una investigación requiere el ejercicio de la creatividad y la inteligencia del investigador, en el momento epistémico de la investigación.

Con respecto a la relación entre el rasgo agradabilidad y actitud hacia la elaboración de tesis, los resultados obtenidos demuestran que no existe correlación entre ambas $(\mathrm{r}=-0.261, \mathrm{p}>0.05)$. Este resultado concuerda también con los hallazgos de Niño de Guzmán, Calderón, y Cassaretto (2003), quienes no encontraron relación entre el rasgo agradabilidad y el rendimiento académico; así también Castro y Martina (2001) hallaron que el rasgo agradabilidad no diferenciaba a dos grupos de estudiantes con diferentes niveles de rendimiento académico. Este hecho podría tener explicación si se reconoce que la dimensión agradabilidad incluye el aspecto humanitario de los hombres, características tales como altruismo, dar afecto y cuidado, preocupación y apoyo emocional (McCrae \& Costa, 1998); por lo tanto, sería evidente que el rasgo agradabilidad implique más relación con el deseo de relacionarse con las personas, que con las predisposiciones de los estudiantes al momento de enfrentar una tesis.

Finalmente, sobre la relación entre el rasgo conciencia y actitud hacia la elaboración de tesis, los resultados obtenidos demuestran que sí existe correlación entre ambas $(\mathrm{r}=0.537, \mathrm{p}<0.05)$. Este hallazgo concuerda también con los resultados obtenidos por Claux (1998), quien encontró influencia y capacidad predictiva en el desempeño escolar por parte del rasgo conciencia. Así también Niño de Guzmán, Calderón y Cassaretto (2003) hallaron asociación positiva entre el rasgo conciencia y el rendimiento académico; de igual forma Castro y Martina (2001) hallaron que el rasgo conciencia diferenciaba a un grupo con mejor rendimiento académico de otro con menor rendimiento académico y, finalmente, Sánchez de Tagle et al. (2010) hallaron que el rasgo conciencia era el que ampliamente diferenciaba a un grupo de estudiantes con mayor promedio académico de otro grupo con menor promedio. Por lo tanto, se demuestra, a través del tiempo, que el rasgo conciencia presenta estabilidad al momento de relacionarse con variables, como el rendimiento académico e inteligencia. Sobre este hecho, una explicación surge al descubrir que la dimensión conciencia evalúa el grado de necesidad de logro, persistencia, competencia, motivación en la conducta y en las metas de una persona (McCrae y Costa, 1998); así también según Colom (2012), el rasgo conciencia influye y propicia el cumplimiento de tareas, alto rendimiento académico y ocupacional, competencia intelectual, metas altas, organización diligente, trabajo duro, manejo del ambiente y solución de problemas, pues las personas con altos niveles en este rasgo se caracterizan por tomarse más tiempo ante las tareas, analizan y proceden cuidadosamente en la resolución de problemas; por lo tanto, es congruente este hallazgo, pues todas las características mencionadas son las que demanda el proceso de elaborar una tesis (Arias, 1998). 
Rasgos de personalidad y actitud hacia la elaboración de tesis en estudiantes del cuarto año de Psicología de la Universidad Peruana Unión - Filial Juliaca

\section{Declaración de financiamiento y de conflicto de intereses:}

El estudio fue financiado por la autora, quien declara no tener algún tipo de conflicto de interés en la investigación realizada.

\section{Correspondencia}

Oscar Mamani Benito

Universidad Peruana Unión-Filial, Juliaca.

Carretera Arequipa Km. 6 - Chullunquiani.

Correo electrónico: psicobenito@gmail.com

\section{REFERENCIAS BIBLIOGRÁFICAS}

Albornoz, M. (2009). El estado de la ciencia 2009. Principales indicadores de ciencia y tecnología Iberoamericanos/Interamericanos. Obtenido de http:// www.vinv.ucr.ac.cr/docs/divulgacion-ciencia/libros-ytesis/el-estado-ciencia-2009.pdf

Ángel Isaza, A., Botero Suárez, H., González, D., Ospina, L., Velasco, M., \& Ocampo, M. (2010). Interés de los estudiantes de medicina por la investigación. CIMEL, Vol. 15, núm. 1. Obtenido de http://www.redalyc.org/ articulo. oa? id $=71720941003$

Arias, F. G. (1998). Mitos y errores en la elaboración de tesis y proyectos de investigación. Caracas: Episteme.

Blanco, N., \& Alvarado, M. (2005). Escala de actitud hacia el proceso de investigación científico social. Revista de Ciencias Sociales, XI (3). Obtenido de http://www. redalyc.org/articulo.oa? $\mathrm{id}=28011311$

Bautista García, V. (2011). Actitud del alumnado de educación secundaria obligatoria frente a la música clásica. Obtenido de http://riuma.uma.es/xmlui/ handle/10630/4893.

Cassaretto Bardales, M. (1999). Adaptación del inventario de personalidad NEO PI-R. Lima: Tesis de pregrado, Pontificia Universidad Católica del Perú.

Cassaretto Bardales, M. (2009). Relación entre las cinco grandes dimensiones de la personalidad y el afrontamiento en estudiantes preuniversitarios de Lima Metropolitana. Lima: Tesis inédita de posgrado en psicología, UNMSM.

Castro Solano, A., \& Martina Casullo, M. (2001). Rasgos de personalidad, bienestar psicológico y rendimiento académico en adolescentes argentinos. Obtenido de http://www.redalyc.org/articulo.oa?id=18011326003.

Claux, M. (1998). School Performance in Peruvian Adolescentes: A Psychological Perspective on Person and Context predictors. Lima: Nijmegen: Mediagroep KUN/AZN.

Colom Marañón, R. (2012). Psicología de las diferencias individuales: teoría y práctica. Madrid: Pirámide.
Costa, P., \& McCrae, R. (1992). Revised NEO Personalyti Inventory (NEO PI-R) and NEO Five-Factor Inventory (NEO-FFI) Professional Manual. Florida: Psychological Assesment Resource, Inc.

Costa, P., \& McCrae, R. (1992). Revised NEO Personalyti Inventory (NEO PI-R) and NEO Five-Factor Inventory (NEO-FFI) Professional Manual. Florida: Psychological Assesment Resource, Inc.

Góngora Prado, M. (2002). El estado, la universidad y el drama de la investigación cientifica. Obtenido de http:// sisbib.unmsm.edu.pe/bibvirtual/publicaciones/escri pensam/2002 n11/estado universidad.htm

Hernández, R., Fernández, C., \& Baptista, P. (2010). Metodología de la investigación científica. México: McGraw Hill.

McCrae, R., \& Costa, P. (1998). Personality Trait Structure as a Human Universal. American Psychologist, 509-516.

Meléndez, L. (2003). La actitud del profesor universitario desde una perspectiva psicológica. Obtenido de http://www.scielo.org.ve/scielo.php?pid=S079897922003000300004\&script=sci_arttext

Miguel, Z., \& Peñaranda, R. (1994). Actitudes de los estudiantes de enfermería de la Universidad Nacional Mayor de San Marcos hacia el curso de investigación y su relación con los factores personales e institucionales. Lima: Tesis inédita de posgrado, UNMSM.

Niño de Guzmán, I., Calderón, A., \& Cassaretto, M. (2003). Personalidad y rendimiento académico en estudiantes universitarios. Revista de Psicología de la Pontifica Universidad Católica del Perú, Volumen XXI (1), 119143.

Portocarrero Maisch, G., \& Bielich Salazar, C. (2006). ¿Por qué los estudiantes no hacen sus tesis? Obtenido de http://test-departamento.pucp.edu.pe/comunicaciones/ images/documentos/cap02-gportocarrero.pdf

Ramos-Rodríguez, M., \& Sotomayor, R. (2008). Realizar o no una tesis: razones de estudiantes de medicina de una universidad pública y factores asociados. Obtenido de http://www.redalyc.org/articulo.oa?id=36311611013 
Rietveldt de Arteaga, F., \& Vera Guadrón, L. (mayoagosto de 2012). Factores que influyen en el proceso de elaboración de la tesis de grado. Zulia, Zulia, Venezuela.

Salinas Fleitman, M. (2005). El papel de la complejidad cognoscitiva y características de personalidad en la formación de actitudes. México D.F.: Tesis de Maestría, Universidad Iberoamericana.

San Martin H., F., \& García P., M. (2006). La tesis y su problemática en la Facultad de Medicina Veterinaria de la UNMSM. Obtenido de http:// www.scielo.org.pe/scielo.php? script=sci arttext\&pid=S1609-91172006000100015

Sánchez de Tagle-Herrera, R., Osornio-Castillo, L., ValadeaNava, S., Heshiki-Nakandakari, L., García-Monroy, L., \& Zárate-Gutiérrez, Z. (2010). Rasgos de personalidad, inteligencia y rendimiento académico en estudiantes de la carrera de médico cirujano. Obtenido de http:// www.redalyc.org/articulo.oa?id=50719083004
Sánchez, S., Collantes, C., Silva, M., Sánchez, J., Aguilar, S., Pérez, M., Córdova, M. (2000). Factores determinantes de la baja producción en investigación cientifica en el Hospital Nacional Dos de Mayo. Lima: Tesis inédita, UNMSM.

Vieytes, R. (2004). Metodología de la investigación en organizaciones, mercado y sociedad: epistemología y técnicas. Buenos Aires: De las Ciencias.

Recibido: 12/03/2014

Aceptado: 10/09/2014 